\title{
Nonconventional Emulsion Polymerization of Styrene with Mixed Anionic and Nonionic Emulsifiers
}

\author{
Shi-Yow LIN, ${ }^{\dagger}$ Ignac CAPEK, ${ }^{*}$ Tien-Jung HsU, and Chorng-Shyan CHERN \\ Department of Chemical Engineering, National Taiwan University of Science and \\ Technology, 43 Keelung Road, Section 4, Taipei 106, Taiwan \\ ${ }^{*}$ Polymer Institute, Slovak Academy of Sciences, 84236 Bratislava, Slovakia
}

(Received March 23, 2000; Accepted July 18, 2000)

\begin{abstract}
Emulsion polymerization of styrene $(\mathrm{St})$ in the presence of mixed anionic/nonionic emulsifiers (SDS/NP $40)$ has been investigated. The polymerization rate $\left(R_{\mathrm{p}}\right)$ vs. conversion $(X)$ curves show a relatively constant $R_{\mathrm{p}}$ region (Smith-Ewart Interval 2) in the range of $X=20-40 \%$ and a shoulder at $X=c a .75 \% . R_{\mathrm{p}}$ is independent of the emulsifier charge composition except the run with SDS or NP 40 as the sole emulsifier. The latex particle size $\left(d_{\mathrm{w}}\right)$ increases with increasing $X$ and the degree of the increased $d_{\mathrm{w}}$ is more pronounced at low $X$. The number of latex particles strongly increases up to $c a .20 \%$ conversion and then remains relatively constant toward the end of polymerization. The particle size distribution of latex particles is quite narrow. The average number of free radicals per particle $(\bar{n})$ is below or close to 0.5 for the polymerization system with SDS alone and mixed SDS/NP 40 emulsifiers before the system experiences the gel effect at high $X$. On the contrary, for the system with NP 40 alone, $\bar{n}$ increases significantly with increasing $X$ and it is well above 0.5 throughout the reaction. The colloidal and kinetic data obtained from this work are discussed in terms of the hairy or close-packed particle (droplet) surface layer, the decreased entry and exit rates of radicals, the relatively high oil-solubility of NP 40, accumulation of polystyrene and NP 40 within the monomer droplets, preservation of monomer droplets during polymerization and formation of double emulsion droplets. The increased uniformity and stability of the initial emulsion may promote the monomer-starved condition even at a relatively low level of $X$.

KEY WORDS Emulsion Polymerization / Mixed Anionic and Nonionic Emulsifiers / Particle Nucleation / Styrene / Double Emulsion Droplets /
\end{abstract}

A mixed emulsifier system comprising anionic and nonionic amphiphiles is widely used for production of commercial polymer latexes. The electrostatic stabilization $^{1,2}$ provided by anionic emulsifier improves latex stability at high temperature, while the steric stabilization ${ }^{3}$ provided by nonionic emulsifier enhances the chemical and freeze-thaw stability of latex products. The latex particles stabilized only by nonionic emulsifier are less stable and show a tendency to flocculate with one another during polymerization in comparison with the recipe containing both anionic and nonionic emulsifiers. ${ }^{4-6}$ Besides, the nonionic emulsifier stabilized particles exhibit a very low affinity towards (poly)electrolytes. ${ }^{7}$

The Smith-Ewart model $\left(R_{\mathrm{p}} \text { or } N_{\mathrm{p}} \propto[E]^{0.6}[I]^{0.4}\right)^{8}$ was examined by emulsion polymerization of hydrophilic and hydrophobic monomers in the presence of mixed anionic/ nonionic emulsifiers. ${ }^{9,10}$ The parameters $R_{\mathrm{p}}, N_{\mathrm{p}},[E]$, and [I] represent the polymerization rate, the number of latex particles per unit volume of water, the emulsifier concentration and the initiator concentration, respectively. The dependencies of the rate of emulsion polymerization of relatively hydrophilic methyl methacrylate on the initiator and emulsifier concentrations $\mathrm{s}^{9,10}$ deviate from those predicted by the Smith-Ewart model. The mixed emulsifiers of sodium dodecyl sulfate (SDS) and nonylphenol polyethoxylate with an average of 40 oxyethylene units per molecule (NP 40) were also used to evaluate the general validity of Smith-Ewart case 2 in emulsion polymerization of styrene. ${ }^{11-13}$ The presence of NP 40 results in the deviation of the reaction kinetics from Smith-Ewart case 2.

Extensive mechanistic studies have led to several mo- dels for the entry of radicals into latex particles and exit of radicals out of latex particles stabilized by ionic emulsifier. ${ }^{14}$ However, the latexes stabilized by the electrosteric mechanism show smaller values of entry $(\rho)$ and exit $\left(k_{\text {des }}\right)$ rate coefficients as compared to the electrostatically stabilized latex particles of the same size. ${ }^{15-16}$ The decreased $\rho$ and $k_{\text {des }}$ in the electrosterically stabilized latexes is ascribed to a hairy layer around the particles, which retards diffusion of oligomeric radicals.

In our previous work ${ }^{13}$ the critical micelle concentration (CMC) in the aqueous solution of mixed SDS/NP 40 emulsifiers was reported to decrease with increasing wt $\%$ NP 40 in the emulsifier charge ([NP 40]). The cloud point for NP 40 in the aqueous phase decreases from 75 to $59^{\circ} \mathrm{C}$ when [NP 40] increases. ${ }^{17}$ The data of particle size and particle size distribution for the styrene emulsion polymerization stabilized only by NP 40 were interpreted in terms of the contribution of miniemulsion polymerization kinetics. ${ }^{17}$ At temperature close to the cloud point of NP $40\left(60^{\circ} \mathrm{C}\right)$, the polyoxyethylene (POE) part of NP 40 is somewhat dehydrated and, therefore, NP 40 molecules can pack into a more dense (or closepacked) emulsifier film on the particle surface. A compact emulsifier film during polymerization leads to collapse of the oil-water interface, in which some NP 40 molecules are rejected from the interface into the aqueous and/or monomer phase. The relatively high oilsolubility of nonionic emulsifier suggests that nonionic emulsifier may act as hydrophobe and this effect increases with increasing temperature. Indeed, addition of NP 40 to the polymerization system of styrene/dodecyl methacrylate/SDS strongly retarded the rate of Ostwald

\footnotetext{
${ }^{\dagger}$ To whom correspondence should be addressed (Tel: + 886-2-2737-6648, Fax: +886-2-2737-6644, E-mail: ling@ch.ntust.edu.tw).
} 
ripening and enhances the droplet stability. ${ }^{18}$

The use of mixed anionic and nonionic emulsifiers offers some interesting studies with respect to partitioning of emulsifier between the monomer and water phases, interaction between anionic and nonionic emulsifiers, extended or contracted conformation of the surface POE chains during emulsion polymerization. The primary objective of this study is therefore to gain a better understanding of the role of NP 40 in the styrene emulsion polymerization stabilized by mixed SDS/NP 40 emulsifiers at temperature below or close to the cloud point of NP 40. Furthermore, the increased uniformity of the initial monomer emulsion droplets and its effect on the polymerization kinetics is investigated.

\section{EXPERIMENTAL}

\section{Materials}

Commercially available styrene monomer (St, Taiwan Styrene Co.) was purified by distillation under reduced pressure twice before use. The reagents sodium persulfate (SPS, Ridel-de Haen), sodium dodecyl sulfate (SDS, 98\% purity, Fluka) and nonylphenol polyethoxylate with an average of 40 oxyethylene units per molecule (NP 40, Fluka) were used as supplied. Deionized water was used throughout this work.

\section{Polymerization Procedure}

Batch emulsion polymerizations were carried out at $60^{\circ} \mathrm{C}$ with the basic recipe comprising $765 \mathrm{~g}$ water, $135 \mathrm{~g}$ $\mathrm{St}$, and $1.4 \mathrm{mM}$ SPS. The composition of the mixture of NP 40 and SDS was varied as shown in Table I. The ingredients of the recipe were charged into the reactor under the nitrogen atmosphere. The mixture was stirred at a constant agitation speed $(400 \mathrm{rpm})$ to form the monomer emulsion. At $60^{\circ} \mathrm{C}$, the average diameter of monomer droplets decreased with stirring time and then leveled off after $1.5 \mathrm{~h}$. Therefore the $\mathrm{St}+$ water + emulsifier mixture was stirred for $2 \mathrm{~h}$ before the addition of initiator to make the initial monomer emulsion more uniform. The monomer conversion was determined by the gravimetric method. The particle size and particle size distribution of the latex samples taken during polymerization were determined by transmission electron microscopy (JEOL TEM-1200 EXII). At least 500 latex particles were measured for each sample. The molecular weight and molecular weight distribution of polystyrene were determined by gel permeation chromatography (Waters 515/2410/Styragel HR 2, HR 4, and HR 6). Other conditions of the polymerization procedure and techniques used have been described in detail elsewhere. ${ }^{21}$

\section{Measurement of Monomer Droplet Size}

During batch emulsion polymerization, samples were sequentially taken and monitored by a video-enhanced microscope (Olympus, BH-2) with magnification $\times 40, \times$ 100 , and $\times 400$ and a resolution of about $500-1000 \mathrm{~nm}$ in diameter. The images of monomer droplets were recorded in the tape and then analyzed to determine the number of droplets per picture and average radius and size distribution of the monomer droplets.
Estimation of Average Number of Radicals per Particle

From the experimental values of the rate of polymerization $\left(R_{\mathrm{p}}\right)$ and number of latex particles per liter water $\left(N_{\mathrm{p}}\right)$, literature values of propagation rate constant $\left(k_{\mathrm{p}}=\right.$ $\left.1.052 \times 10^{9} \exp \left(-5052.3 \mathrm{~T}^{-1}\right) \mathrm{dm}^{3} \mathrm{~mol}^{-1} \mathrm{~s}^{-1}\right)^{22}$ and equilibrium concentration of $\mathrm{St}$ in the latex particles $\left([M]_{\mathrm{p}}=\right.$ $\left.5.2 \mathrm{~mol} \mathrm{dm}{ }^{-3}\right),{ }^{23}$ the average number of free radicals per particle $(\bar{n})$ was estimated by the following equation:

$$
R_{\mathrm{p}}=k_{\mathrm{p}}[M]_{\mathrm{p}}\left(\bar{n} N_{\mathrm{p}} / N_{\mathrm{A}}\right)
$$

where $N_{\mathrm{A}}$ is Avogadro number.

\section{RESULTS}

The S-shaped monomer conversion $(X)$ vs. time $(t)$ curves, typical for the St emulsion polymerization with various emulsifier compositions, are shown in Figure 1. The data of $R_{\mathrm{p}}$ as a function of $X$ and emulsifier feed composition are illustrated in Figure 2. The reproducibility of the kinetic data of $X, R_{\mathrm{p}}, d_{\mathrm{w}}$, and $N_{\mathrm{p}}$ was verified by the polymerization stabilized by SDS alone, as shown in our previous work. ${ }^{21}$ The parameter $d_{\mathrm{w}}$ is the weight average polymer particle diameter. In the polymerization stabilized only by SDS, the region of the relatively constant $R_{\mathrm{p}}$ (Interval 2) is in the range of $X=20-40 \%$. The maximal rate is located at $X=c a$. $30--35 \%$, and the shoulder at $X=c a .70-80 \%$. In the polymerizations with mixed SDS/NP 40 emulsifiers, the non-distinct Interval 2 also ranges roughly from 20 to $40 \%$ conversion. Note that all the polymerizations with mixed SDS/NP 40 emulsifiers show very similar $R_{\mathrm{p}}$ vs. $X$ profiles (Figure 2 b). By contrast, in the polymerization with NP 40 only, $R_{\mathrm{p}}$ increases slightly with increasing $X$ during the first $50 \%$ conversion.

The time evolution of monomer droplets during polymerization was followed and the data are shown in Figures 3 and 4 . The monomer droplets were still observed at high $X$ (up to $c a .95 \%$ ) in both polymerizations with SDS alone ${ }^{21}$ and mixed SDS/NP 40 emulsifiers (Figures 3 and 4 ). It should be noted that the concentration of SDS or NP 40 is well above its CMC in all polymerizations (Table I). The rapidly increased $N_{\mathrm{p}}$ before $X=10 \%$ is observed for both the polymerizations with mixed SDS/NP 40 emulsifiers and SDS alone (Figure 5). The variation of $d_{\mathrm{w}}$ with the emulsifier type and conversion was shown in Figure 6 . The dried particle size increases with increasing $X$ and the degree of the increased $d_{\mathrm{w}}$ with $X$ is much more pronounced for the system stabilized by NP 40 alone. In addition, the increase of $d_{\mathrm{w}}$ is more pronounced below $10 \%$ conversion.

Figure 7 shows the dependence of $R_{\mathrm{pc}}$, determined during the relatively constant rate period, on the emulsifier charge composition (SDS/NP 40). The polymerization with NP 40 alone is very slow $\left(R_{\mathrm{pc}}=c a .1 \mathrm{mM}\right.$ $\min ^{-1}$, Figure $2 a$ ). The addition of a small amount of SDS into the reaction system, however, abruptly increases $R_{\mathrm{pc}}$. Further increasing the level of SDS in the emulsifier charge does not affect $R_{\mathrm{pc}}$ until a very high amount of SDS in the emulsifier mixture (SDS/NP 40> $3 / 1$ ) is reached. On the contrary, the addition of only a small amount of SDS to the reaction mixture (e.g., SDS/ NP $40=1 / 2$ ) strongly increases $N_{\mathrm{p}}$ (Figure 8 ). 


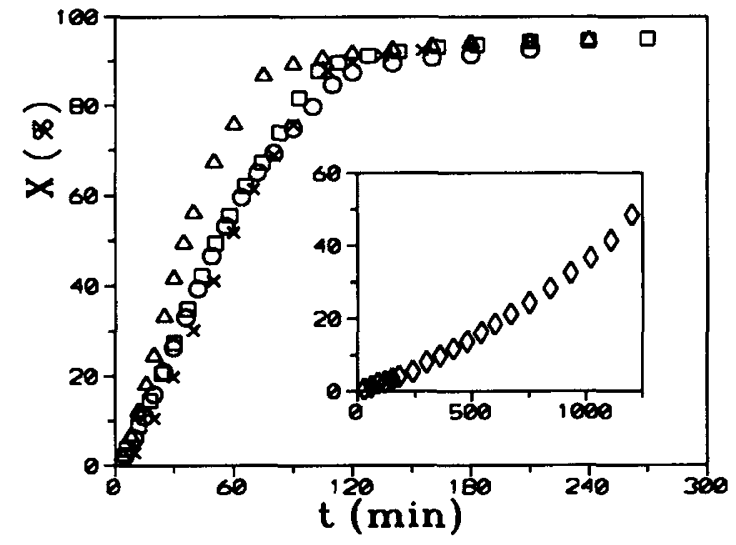

Figure 1. Variation of monomer conversion with time in the $\mathrm{St}$ emulsion polymerization at different ratios of SDS/NP 40 (see Table I). SDS/NP $40=(\triangle) 1 / 0,(\bigcirc) 3 / 1,(\square) 1 / 1,(\times) 1 / 2,(\diamond) 0 / 1$.

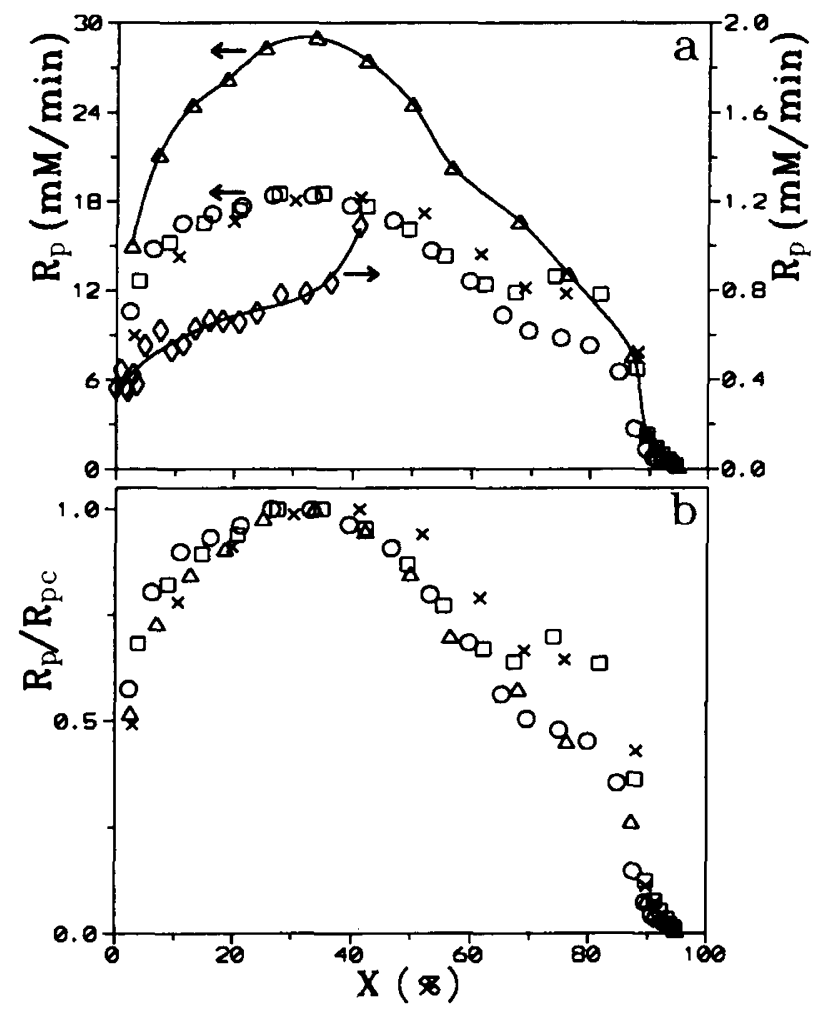

Figure 2. Variation of polymerization rate with monomer con version in the $\mathrm{St}$ emulsion polymerization at different ratios of $\operatorname{SDS} / \mathrm{NP} 40 . \mathrm{SDS} / \mathrm{NP} 40=(\triangle) 1 / 0,(\bigcirc) 3 / 1,(\square) 1 / 1,(\times) 1 / 2,(\diamond) 0 / 1$

From the $R_{\mathrm{p}}$ and $N_{\mathrm{p}}$ data, $\bar{n}$ was calculated as a function of $X$ and the composition of the emulsifier mixture (Figure 9). In the polymerization with mixed SDS/NP 40 emulsifiers, $\bar{n}$ remains relatively constant during polymerization except at high $X$ (Figure 9 ). That is, $\bar{n}$ is below or close to 0.5 before the system experiences a significant gel effect at high $X$. On the contrary, for the polymerization with NP 40 alone, $\bar{n}$ increases significantly with increasing $X$ and it is well above 0.5 throughout the reaction.

In the polymerization stabilized only by SDS or NP 40 , the polydispersity index $\left(\mathrm{PDI}=d_{\mathrm{w}} / d_{\mathrm{n}}\right)$ of the latex particle size distribution is somewhat broad at very low and very high $X$ (Figure 10). The parameter $d_{n}$ represents
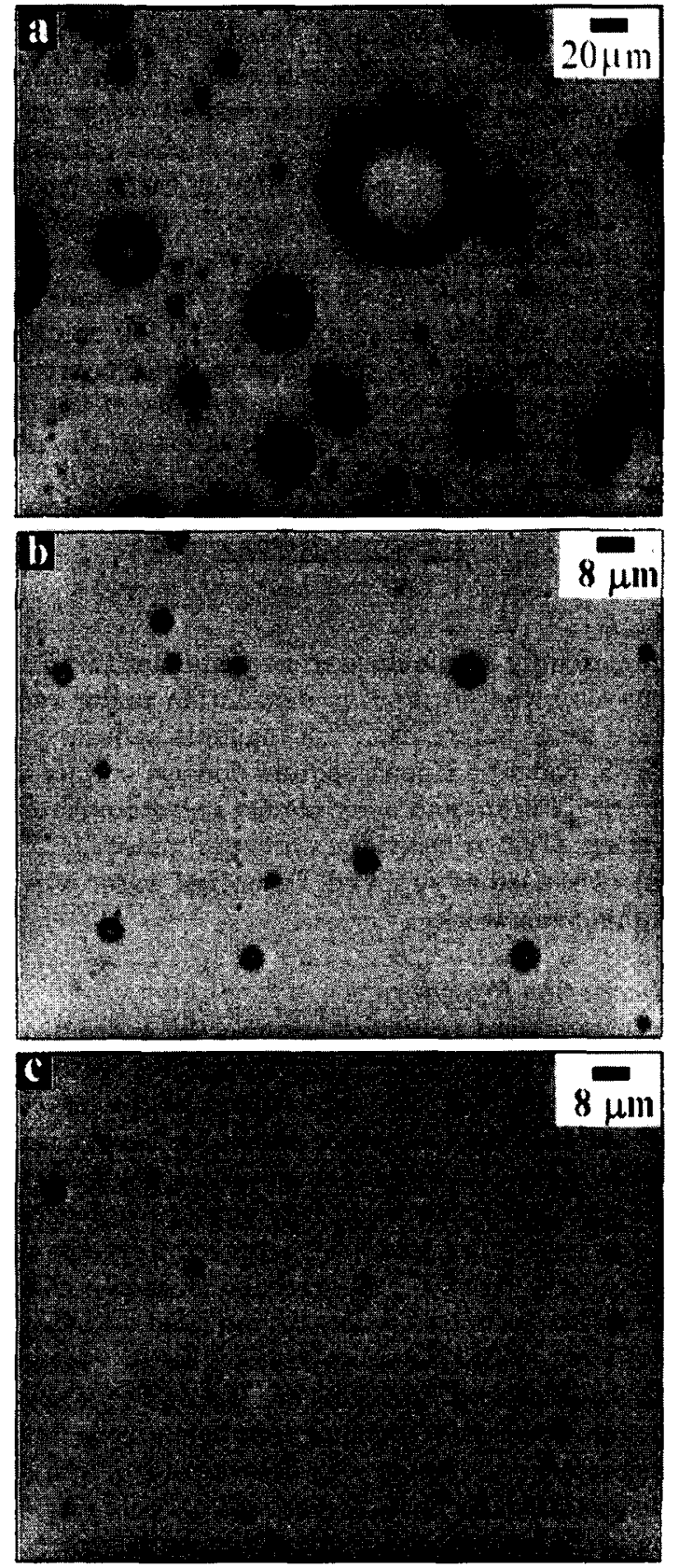

Figure 3. Representative images of monomer droplets at $X=$ (a) $19.9 \%$, (b) $51.8 \%$, and (c) $68.9 \%$ in the St emulsion polymerization at $\operatorname{SDS} / \mathrm{NP} 40=1 / 2$.

the number average particle diameter. As expected, PDI decreases with increasing $X$ since the residence time distribution of the growing particles becomes narrower as the batch polymerization proceeds.

The use of the video-enhanced microscope confirms the presence of the water-in-oil-in-water (w/o/w) double emulsion droplets for all five recipes used in this work. Figure 11 shows two representative images of double emulsion droplets at different levels of $X$ for the polymerization system with various ratios of SDS/NP 40. The two aqueous phases, the one contained in the oil drops (as indicated by the arrow in Figure 11) and the continuous phase, are separated by the monomer phase, which acts just like a permeable barrier for the reactants. The reason for the formation of $\mathrm{w} / \mathrm{o} / \mathrm{w}$ double emulsion drop- 
Table I. Recipe used to prepare styrene latex particles with various emulsifier compositions

\begin{tabular}{|c|c|c|c|c|c|}
\hline \multirow{2}{*}{ SDS/NP 40} & [SDS] & [NP 40] & $N_{\mathrm{m}}{ }^{\mathrm{a}}$ & $f_{\mathrm{m}}{ }^{\mathrm{b}}$ & wt ratio \\
\hline & $10^{-3} \mathrm{~mol} \mathrm{dm}^{-3}$ & $10^{-4} \mathrm{~mol} \mathrm{dm}^{-3}$ & $10^{-4} \mathrm{~mol} \mathrm{dm}^{-3}$ & SDS:NP 40 & SDS:NP 40 \\
\hline $1 / 0$ & 18.2 & - & 1.12 & $1: 0$ & $100: 0$ \\
\hline $3 / 1$ & 13.6 & 2.94 & 1.12 & $3: 1$ & $97.9: 2.1$ \\
\hline $1 / 1$ & 9.09 & 5.88 & 1.12 & $1: 1$ & 93.9:6.1 \\
\hline $1 / 2$ & 6.60 & 7.84 & 1.12 & $1: 2$ & $89.4: 10.6$ \\
\hline $0 / 1$ & - & 11.8 & 1.12 & $0: 1$ & $0: 100$ \\
\hline
\end{tabular}

${ }^{a} N_{\mathrm{m}}$ is the number of micelles per liter estimated from the equation $N_{\mathrm{m}}=([E]-\mathrm{CMC}) / \mathrm{m}$, where $[E]$ is the emulsifier (SDS or NP 40) concentration, CMC is $1.02 \times 10^{-2} \mathrm{~mol} \mathrm{dm}{ }^{-3}$ for SDS and $2.91 \times 10^{-4} \mathrm{~mol} \mathrm{dm}{ }^{-3}$ for NP 40 at $60^{\circ} \mathrm{C}$, and $\mathrm{m}$ is the aggregation number of one micelle ( 71 for SDS and 7.88 for NP 40). ${ }^{19,20}{ }^{\mathrm{b}}{ }^{\mathrm{b}} \mathrm{f}_{\mathrm{m}}$ is the ratio of SDS micelles to NP 40 micelles, with the assumption that there exist two different micelles (for SDS and NP 40, respectively).

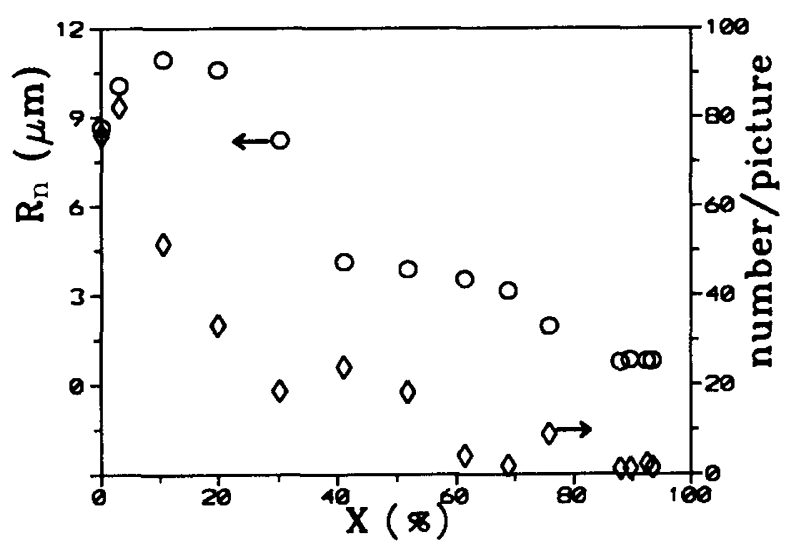

Figure 4. Variation of number average monomer droplet size and number of monomer droplets per picture $(406 \times 289 \mu \mathrm{m})$ with monomer conversion in the St emulsion polymerization at SDS/NP $40=1 / 2$.

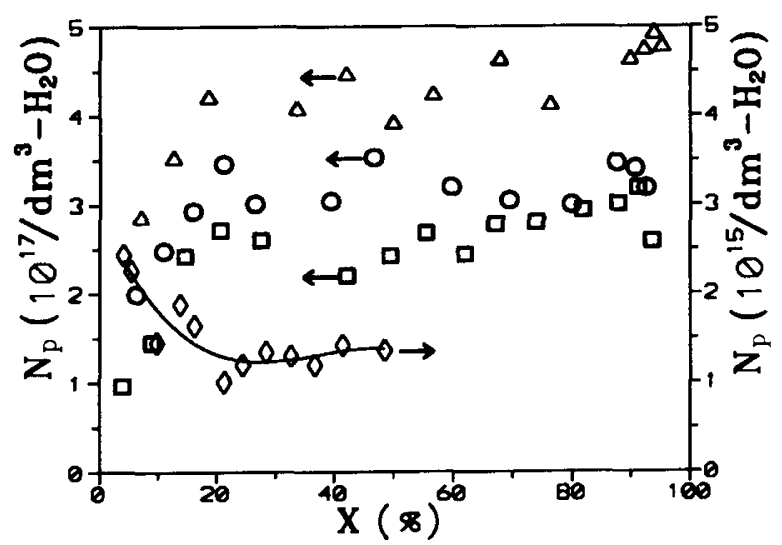

Figure 5. Variation of latex particle number with monomer conversion in the St emulsion polymerization at different ratios of SDS/NP 40. SDS/NP $40=(\triangle) 1 / 0,(\bigcirc) 3 / 1,(\square) 1 / 1,(\diamond) 0 / 1$.

lets is not clear at this time, but this may also contribute to the observed polymerization kinetics in addition to the well known features of the Smith-Ewart model.

\section{DISCUSSION}

\section{Polymerization Kinetics}

In the polymerization stabilized by SDS alone or mixed SDS/NP 40 emulsifiers, the $R_{\mathrm{p}} v s . X$ data exhibiting one maximum and one shoulder (Figure 2) deviate from the micellar nucleation model. ${ }^{8}$ Furthermore, the increased fraction of SDS in the emulsifier mixture is expected to increase $N_{\mathrm{p}}$ and $R_{\mathrm{p}}$ (proportional to $N_{\mathrm{p}}$ ) as well.

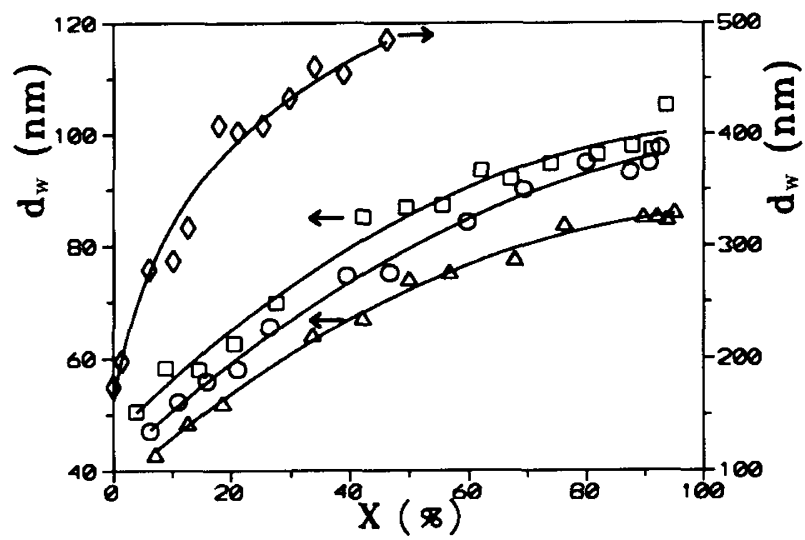

Figure 6. Variation of weight average particle size with monomer conversion in the St emulsion polymerization at different ratios of SDS/NP $40 . \operatorname{SDS} / \mathrm{NP} 40=(\triangle) 1 / 0,(\bigcirc) 3 / 1,(\square) 1 / 1,(\diamond) 0 / 1$.

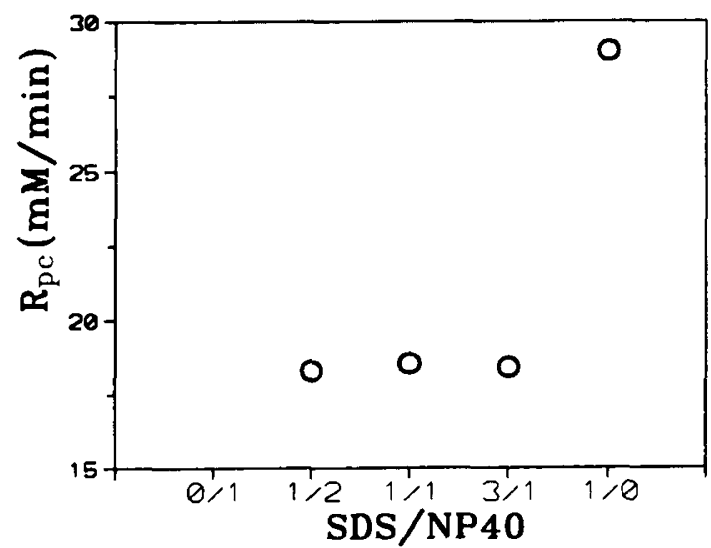

Figure 7. Variation of polymerization rate determined during the relatively constant reaction rate period $\left(R_{\mathrm{pc}}\right)$ at different ratios of SDS/NP 40 in the St emulsion polymerization

However, the $R_{\mathrm{pc}}$ and $N_{\mathrm{p}}$ data in Figures 7 and 8 show that this is not the case. The rapidly increased $R_{\mathrm{pc}}$ with SDS/NP 40 being increased from $0 / 1$ to $1 / 2$ may result from the fact that SDS acts as the major particle generator while NP 40 only serves as an auxiliary stabilizer in emulsion polymerization. ${ }^{24}$

The stationary Interval 2 for the polymerization of hydrophobic monomer such as St ends at $X=c a .40-50 \%$ as a result of the disappearance of monomer droplets. The presence of monomer droplets at high $X$ (up to $c a$. $95 \%)$ in both polymerizations with SDS alone ${ }^{21}$ and mixed SDS/NP 40 emulsifiers (Figures 3 and 4) is not completely consistent with the classical emulsion polym- 


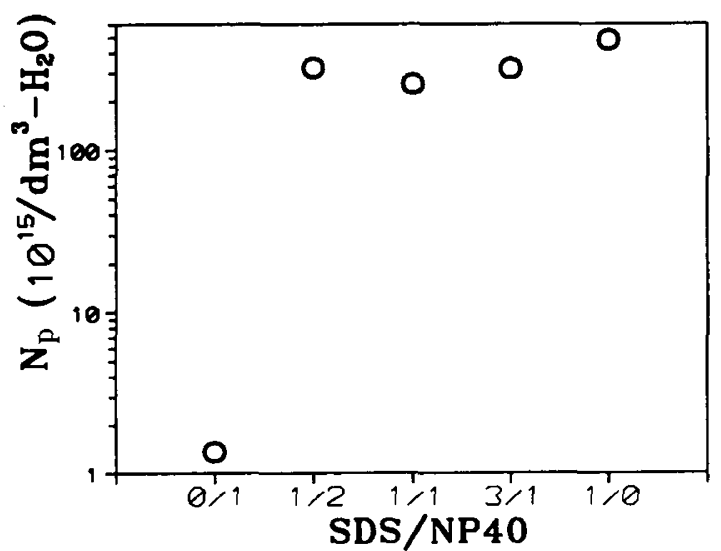

Figure 8. Number of latex particles at different ratios of SDS/NP 40 in the St emulsion polymerization.

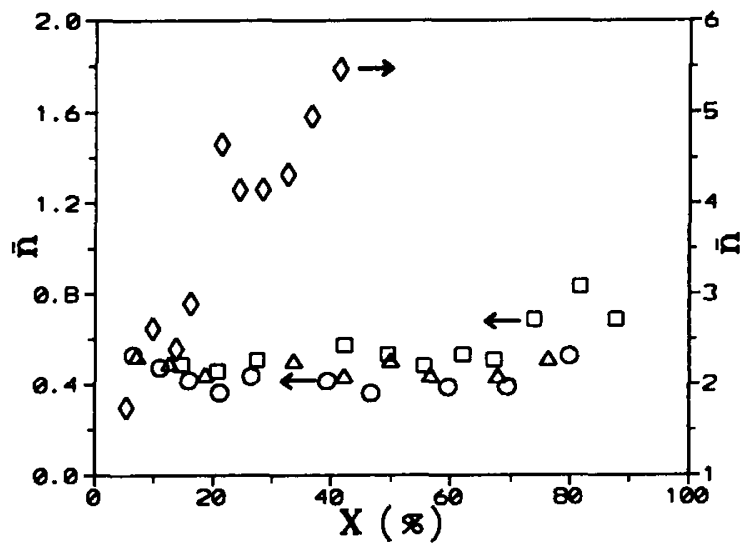

Figure 9. Variation of average number of free radicals per particle with monomer conversion in the St emulsion polymerization at different ratios of SDS/NP 40. SDS/NP $40=(\triangle) 1 / 0,(\bigcirc) 3 / 1,(\square) 1 /$ $1,(\diamond) 0 / 1$.

erization mechanism. Both the size and number of monomer droplets decrease with increasing $X$ and the degree of these variations is the most significant below $30 \%$ conversion. These data indicate that diffusional degradation of monomer droplets is greatly suppressed after $X=c a$. $30 \%$. This is because droplets act mainly as the monomer reservoir at the beginning of polymerization. The shrinkage of droplets is very fast before $X=$ $30 \%$ in order to supply the growing latex particles with monomer. After then, both the size and number of droplets only decrease gradually with increasing $X$, which is attributed to the increased stability of droplets caused by the accumulation of hydrophobic molecules (polystyrene (PSt), NP 40, etc.) in the monomer droplets.

The rapidly increased $N_{p}$ before $X=10 \%$ for both the polymerizations with mixed SDS/NP 40 emulsifiers and SDS alone (Figure 5) is due to the fact that SDS or NP 40 is well above its CMC and there is a large population of micelles available for nucleation (Table I). The maximal $R_{\mathrm{p}}$ is the result of the rapidly increased $N_{\mathrm{p}}$ with $X$. After the maximum is reached, $R_{\mathrm{p}}$ starts to decrease with increasing $X$. Note that monomer droplets are still present in the reaction system even beyond the SmithEwart Interval $2(X>50 \%)$, as shown in Figures 3 and 4. It is therefore postulated that the decreased $[M]_{\mathrm{p}}$ with $X$

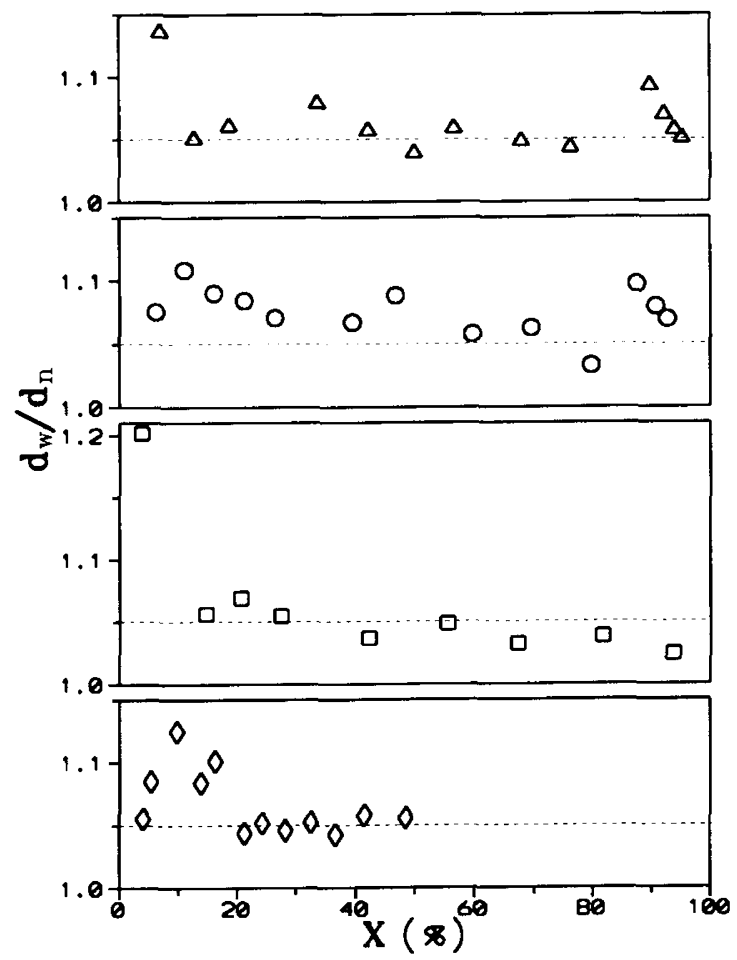

Figure 10. Variation of polydispersity index of the particle size distribution with monomer conversion in the St emulsion polymerization at different ratios of SDS/NP $40 . \operatorname{SDS} / \mathrm{NP} 40=(\triangle) 1 / 0$, (○) $3 / 1,(\square) 1 / 1,(\diamond) 0 / 1$

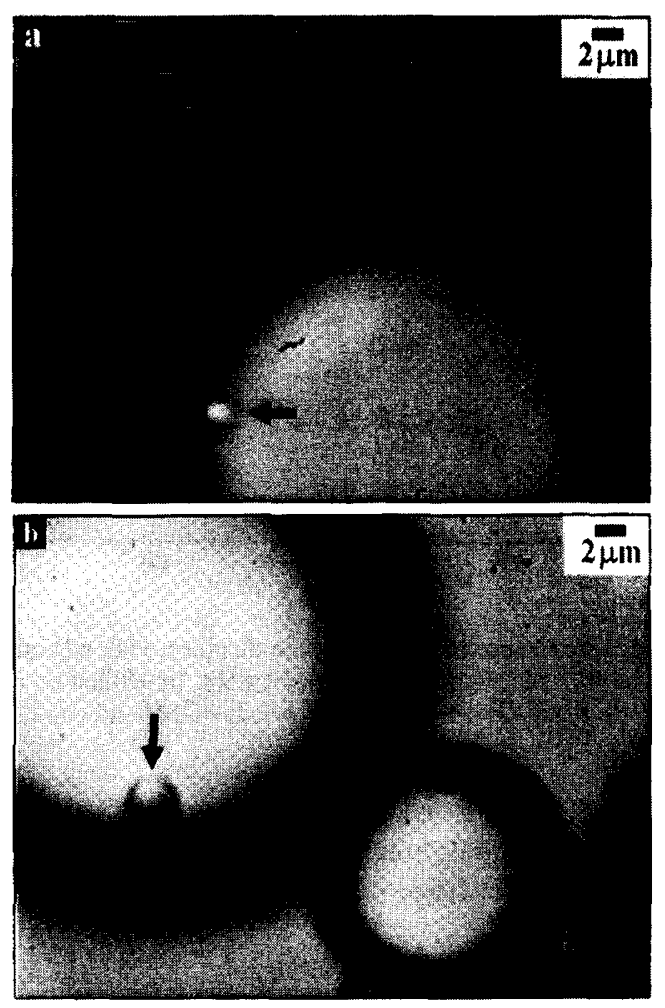

Figure 11. Representative images of double emulsion droplets for the ratio of SDS/NP $40=(a) 1 / 2(X=10.6 \%)$ and (b) $3 / 1(X=$ $51.8 \%$ ). 
is responsible for the decrease of $R_{\mathrm{p}}$ at $X=30-50 \%$ and the polymerization proceeds under the apparent monomer-starved condition (monomer droplets are still present in the reaction system but the ratio of monomer to polymer in latex particles is very low $\left.(c a .0 .15)^{25}\right)$. The relatively low monomer concentration at the reaction loci results from the decreased monomer transfer rate from droplets to latex particles and dilution of monomer in these particles by the hydrophobic chains of mixed emulsifiers (both SDS and NP 40) ${ }^{26}$ and the dissolved NP $40 .{ }^{27}$ Besides, chain transfer of radicals to the dissolved NP 40 (the degradative chain transfer) may also contribute to the reduction in $R_{\mathrm{p}}{ }^{28}$ Thus, the presence of monomer droplets at high $X$ should be related to the depressed transfer of monomer from droplets to latex particles. This may explain why a relatively narrow Interval 2 was observed in the polymerizations investigated in this work.

Note that the rate shoulder in the polymerization with SDS alone or mixed SDS/NP 40 emulsifiers with a lower level of NP 40 is transformed to a distinct maximum in the polymerizations with a higher level of NP 40 (Figure 2). A similar behavior was observed in the St emulsion polymerizations stabilized only by NP $40 .{ }^{17}$ The appearance of the rate shoulder at $X=70-80 \%$ is simply due to the accumulation of radicals in latex particles (the gel effect). Another possible explanation is the variation of the oil-water interfacial properties during polymerization. The particle surface layer is extended due to the adsorption of NP 40 released from the monomer phase during polymerization. The hairy particle surface layer decreases the exit rate coefficient $\left(k_{\text {des }}\right) .{ }^{15}$ Indeed, the slight differences in $d_{\mathrm{w}}$ and $N_{\mathrm{p}}$ for the polymerizations with SDS/NP $40=1 / 0,1 / 1$, and $3 / 1$ (Figures 5 and 6) imply that the difference in the rate shoulder should be interpreted in terms of the different structures of the particlewater interface (see the discussion shown later).

The value of $R_{\mathrm{pc}}$ for the polymerization with NP 40 alone is very small $\left(R_{\mathrm{pc}}=c a .1 \mathrm{mM} \mathrm{min}^{-1}\right.$, Figure $\left.2 \mathrm{a}\right)$. Figure 7 shows that the addition of a small amount of SDS into the reaction system, however, abruptly increases $R_{\mathrm{pc}}$. The first abrupt increase in $R_{\mathrm{pc}}$ is attributed to transformation of the steric stabilization mechanism (SDS/NP $40=0 / 1$ ) to the electrosteric stabilization mechanism (SDS/NP 40=3/1). In the former case, large latex particles (or a small population of particles) are produced. The slow polymerization stabilized by NP 40 is attributed to the agglomeration of latex particles, the small total particle surface area and the pseudo-bulk kinetics. Figures 5 and 6 indicate an initial abrupt increase in $N_{\mathrm{p}}(X \leq 5 \%)$, followed by a rapid decrease in $N_{\mathrm{p}}$ (or an increase in $d_{\mathrm{w}}$ ) for the polymerization with SDS/ NP $40=0 / 1$, which is typical for the coagulative nucleation mechanism. ${ }^{14}$ Thus, for the polymerization with SDS/NP $40=0 / 1, N_{\mathrm{p}}$ varies significantly with $X$ and agglomeration and nucleation of latex particles take place simultaneously during polymerization. When $X$ is below ca. $20 \%$, latex particles grow by coagulation, which is strongly competitive with the propagation reaction in latex particles. At high $X$ (above ca. 20\%), however, growth of the more stable latex particles by polymerization of the imbibed monomer becomes more important. For the polymerizations with SDS/NP $40=1 / 2,1 / 1,3 / 1$, or $1 / 0$, by contrast, the much larger number of latex particles and the radical compartmentalization effect result in the increased $R_{\text {pc. }}$.

The relatively constant $R_{\mathrm{pc}}$ (Figure 7) and $N_{\mathrm{p}}$ (Figure 8) for the polymerizations with a wide range of SDS/ NP 40 deviate from the theoretical prediction (see the above discussion). This is most likely due to the differences in the nature of SDS and NP 40 used in the St emulsion polymerization and the particle surface layer produced. Smith-Ewart case 2 (i.e., $N_{\mathrm{p}} \propto[E]^{0.6}[I]^{0.4} \mathrm{ob-}$ tained from the polymerization with mixed SDS/NP 40 emulsifiers at $80^{\circ} \mathrm{C}$ ) is more applicable to the polymerization stabilized only by $\mathrm{SDS}^{11,12}$ However, for the polymerization carried out at $60^{\circ} \mathrm{C}$, the relationship $N_{\mathrm{p}} \propto$ $[E]^{0.6}$ can be observed when the level of NP 40 in the emulsifier mixture increases up to $2.1 \mathrm{wt} \%$ (SDS/NP 40 $=3 / 1$, Table I). The effect of NP 40 on the polymerization kinetics (or the degree of deviation from the micellar nucleation model) increases with increasing temperature. The contracted conformation of the surface POE chains and the relatively high oil-solubility of NP 40 at $80^{\circ} \mathrm{C}$ (the cloud point of NP $40=c a .72^{\circ} \mathrm{C}^{29}$ ) indicate that $\mathrm{NP}$ 40 also acts as a hydrophobe in the St emulsion polymerization. On the other hand, the amphiphilic properties of NP 40 is maintained at $60^{\circ} \mathrm{C}$ and, therefore, NP 40 can take part in the modification of the particle-water interface via the mutual interaction between SDS and NP 40 . This will then increase the density of the particle-water interfacial layer significantly. Indeed, the close-packed structure of the particle-water interface produced via the formation of complex between SDS and the nonionic POE type emulsifier was reported in the literature. ${ }^{30,31}$

Figure 10 shows that the polymer particles are relatively monodisperse except the latex sample taken during the initial stage of polymerization. To form monodisperse polymer particles, the particle nucleation period should be very short, all the oligomeric radicals generated in the continuous aqueous phase should be captured by the existing latex particles before they precipitate out of the continuous phase and form new particle nuclei, and the coalescence of premature particles should be prevented in the particle growth stage. In this study, however, the particle nucleation period is relatively long (up to ca. $20 \%$ conversion). The increased $N_{\mathrm{p}}$ with $X$ and the narrow particle size distribution (PSD) are observed in the miniemulsion polymerization, that is, the partial release of monomer from monomer droplets allows the additional particle growth and the preserved monomer droplets promotes monomer droplet nucleation. This is supported by the fact that the number average radius of monomer droplets decreases with increasing $X$, but a significant population of tiny monomer droplets is still present toward the end of polymerization (Figure 4). However, the mixed modes of particle nucleation (micellar nucleation/monomer droplet nucleation) would lead to the bimodal or broad PSD. The absence of significant monomer droplet nucleation may be explained as follows. The very large total particle surface area due to the initial abrupt increase in $N_{\mathrm{p}}(X \leq 5 \%) \mathrm{im}$ plies the retarded secondary particle nucleation in the subsequent polymerization, that is, the initiating radicals are preferentially captured by latex particles. The close-packed droplet surface layer not only depresses the 
monomer droplet degradation but also reduces the radical entry rate. ${ }^{32,33}$ Furthermore, the continuous release of NP 40 (or SDS from double emulsion droplets, see the discussion shown below) from monomer droplets is mainly used to increase the stability of latex particles and, therefore, the additional particle nucleation beyond $20 \%$ conversion is prohibited. In addition, the apparent monomer-starved condition caused by the relatively stable monomer droplets disfavors the saturation of $\mathrm{mi}$ celles (if present) by monomer. Thus, the entry of radicals into such a micelle may not lead to the effective particle nucleation. This is further supported by the St emulsion polymerization stabilized only by NP 40, where the PSD of the latex product is narrow $\left(N_{\mathrm{p}}\right.$ is kept relatively constant above $20 \%$ conversion) and the released NP 40 species are solely used for stabilization of the growing latex particles. ${ }^{17}$ Thus, the contribution of monomer droplets to the whole particle nucleation mechanism is not significant. As discussed above, the very large population of latex particles and relatively stable monomer droplets may promote the apparent monomerstarved condition and contribute to the evolution of PSD. The depressed formation of long-chain oligomer to form particle precursors in water and saturation of micelles by monomer do not promote the secondary particle nucleation. As a result, $N_{\mathrm{p}}$ remains relatively constant above $20 \%$ conversion for the polymerizations with mixed SDS/NP 40 emulsifiers and SDS alone. Indeed, the monomer-starved condition retards the secondary particle nucleation and, thereby, favors the formation of latexes with higher uniformity. ${ }^{34}$ Furthermore, the competitive growth of latex particles may also increase the uniformity of PSD. ${ }^{35}$ However, future research is required to clarify the above postulations.

The weight average molecular weight of the latex sample taken at the end of polymerization $\left(M_{\mathrm{w}}\right)$ is $8.48 \times$ $10^{5}, 7.41 \times 10^{5}, 8.00 \times 10^{5}$, and $6.37 \times 10^{5}$, respectively, for the runs with SDS/NP $40=1 / 0\left(d_{\mathrm{w}}=86.1 \mathrm{~nm}\right), 1 / 1\left(d_{\mathrm{w}}\right.$ $=97.6 \mathrm{~nm}), 2 / 1\left(d_{\mathrm{w}}=105.2 \mathrm{~nm}\right)$, and $0 / 1\left(d_{\mathrm{w}}=484.8 \mathrm{~nm}\right)$. As expected, $M_{\mathrm{w}}$ increases with decreasing $d_{\mathrm{w}}$ (or increasing $N_{\mathrm{p}}$ ) due to the increased degree of segregation of radicals among the latex particles. The relationship $M_{\mathrm{w}}=1.502 \times 10^{6} d_{\mathrm{w}}{ }^{-0.139}$ is thus established. No correlation between the polydispersity index of the molecular weight distribution $\left(M_{\mathrm{w}} / M_{\mathrm{n}}\right)$ and the emulsifier charge composition is observed, where $M_{\mathrm{n}}$ is the number average molecular weight. The average value of $M_{\mathrm{w}} / M_{\mathrm{n}}$ is $1.77 \pm 0.19$ in this series of experiments.

\section{Structured Latex Particle Surface Layer}

In the St emulsion polymerization stabilized by mixed SDS/NP 40 emulsifiers, the close-packed or hairy particle surface layer results from the mutual interaction between SDS and NP 40 at the particle-water interface and accumulation of the relatively hydrophobic NP 40 molecules at the inner side of the particle surface layer. NP 40 can increase the viscosity of the particle surface layer, thereby leading to a reduction in the mass transfer of monomeric radicals (exiting) and oligomeric radicals (entering) through this interfacial region. ${ }^{15}$ The continuous evolution of the particle-water interfacial layer is one of the major reasons why the constant $R_{\mathrm{p}}$ interval is quite short ( $X=20-40 \%$, Figure 2). The rather thick hairy particle surface layer formed by NP 40 alone is transformed into the less thick close-packed particle (droplet) surface layer by the addition of SDS. This interfacial layer acts as a barrier to the entering radicals and, thus, slows down the polymerization. ${ }^{32,33}$ Although the viscosity in the close-packed region of SDS/NP 40 is higher than that in the region comprising the extended POE chains of NP 40, the radical should have a shorter distance to diffuse through the close-packed interfacial zone. Thus, it is reasonable to assume that the total time for the radical to diffuse through such a close-packed particle surface layer would be less than that through the extended POE chains. ${ }^{15}$ In favor of such a closepacked particle-water interface is that the data of $d_{\mathrm{w}}$ or $N_{\mathrm{p}}$ are quite similar for the polymerizations with mixed SDS/NP 40 emulsifiers (Figure 8). Similar results were also observed in our previous reports. ${ }^{11,12}$

For the polymerizations stabilized only by SDS and mixed SDS/NP 40 emulsifiers, the rapidly increased $N_{\mathrm{p}}$ with $X$ (up to $20 \%$ ) is primarily due to the micellar particle nucleation mechanism (Figure 5). Indeed, monomer droplets act mainly as the monomer reservoir at the beginning of polymerization and, hence, the most significant decrease in both the size and number of monomer droplets is observed below 30\% conversion (Figure 4). The subsequent slight increase in $N_{\mathrm{p}}(X \geq 20 \%)$ can be attributed to nucleation in the remaining micelles and/or minor contribution of miniemulsion polymerization (the increased stability of monomer droplets due to the accumulation of PSt). ${ }^{32,36}$

The value of $d_{\mathrm{w}}$ increases abruptly with increasing $X$ and the increase of $d_{\mathrm{w}}$ below $20 \%$ conversion is the most pronounced for the polymerization stabilized only by NP 40 (Figure 6). The $d_{\mathrm{w}} v s$. $X$ profile for the NP 40 stabilized polymerization has been interpreted in terms of the coagulative nucleation mechanism (see the above discussion). A less pronounced increase in $d_{\mathrm{w}}$ is observed for the polymerizations with SDS alone and mixed SDS/NP 40 emulsifiers. According to the micellar nucleation model, $N_{\mathrm{p}}$ remains relatively constant with increasing $X$ except the $N_{\mathrm{p}}$ data determined in the initial stage of polymerization. This study also indicates that $d_{\mathrm{w}}$ increases with increasing $X$ in Interval 3, which is inconsistent with the theoretical prediction that the particle size remains relatively constant at high $X$. The slight increase of $d_{\mathrm{w}}$ at very high $X$ can be attributed to diffusion of monomer from droplets to the still growing latex particles and/or limited flocculation of latex particles. The deviation of the kinetic data from the micellar nucleation model is attributed to the preservation of monomer droplets (i.e., the decreased mass transfer rate).

The events of entry/exit/re-entry (3E) of radicals govern the fine emulsion polymerization kinetics. ${ }^{14}$ The $3 \mathrm{E}$ events may be operative in the polymerization with SDS alone, in which fine latex particles are produced. The rather dense particle surface layer formed by SDS and NP 40 reduces the entry and exit rates of radicals. ${ }^{30,32,36}$ It is then postulated that the entry of the surface active oligomeric radicals into latex particles is hindered to a larger extent than the exit of monomeric radicals out of the particles due to the differences in the charge and molecular weight of these radicals involved. As a result, $\bar{n}$ is slightly below 0.5 (Figure 9). This is, however, not the 
case for the latex particles stabilized by SDS alone, where $\bar{n}$ is governed by desorption of radicals out of the latex particles. ${ }^{15}$ Furthermore, $\bar{n}$ is independent of $X$ for the polymerizations with SDS/NP 40 except in the high $X$ region (Figure 9). Accumulation of radicals at high $X$ is the result of the rapidly increased viscosity inside the latex particles, which leads to the rate shoulder (the gel effect). This also can be interpreted in terms of the shift in the emulsifier composition at the particle-water interfacial layer during polymerization, leading to the variation of the radical entry and exit processes. At the beginning of polymerization, a large fraction of NP 40 is dissolved in the monomer phase and the interface is rich in SDS. As the polymerization advances, NP 40 can be released from the monomer phase and penetrates into the interfacial layer. This will then make the particle surface layer rich in NP 40, which reduces the radical entry and exit rates. For the polymerization with NP 40 alone, $\bar{n}$ increases significantly with increasing $X$ and it is well above 0.5 throughout the reaction. This is attributed to the coagulative particle nucleation mechanism. Large latex particles may also absorb small, active particle precursors and this will then lead to the particle growth and the increase of $\bar{n}$. In addition, the viscous latex particles and the hairy particle surface layer may result in a decrease in the diffusion coefficient of radicals, leading to the reduced $k_{\text {des }}$ (or the increased $\bar{n}$ ). Indeed, the $k_{\text {des }}$ value for the latex particles stabilized by NP 30 is one order of magnitude smaller than that for the particles stabilized by ionic emulsifier. ${ }^{16}$ Thus, the exit of radicals from the latex particles stabilized by NP 40 is the ratedetermining step. This behavior was also interpreted in terms of the pseudo-bulk polymerization kinetics starting from $5 \%$ conversion, at which point $d_{\mathrm{w}}$ is around 200 $\mathrm{nm}$, large enough to accommodate more than one radical in the particle (Figure 6) ${ }^{17}$ In addition, the reaction temperature $\left(60^{\circ} \mathrm{C}\right)$ is well below the glass transition temperature of PSt $\left(105^{\circ} \mathrm{C}\right)$ and the flocculated, monomerstarved polymer particle is viscous enough to retard the bimolecular termination of radicals. ${ }^{17,37}$

The double emulsion droplets were not only observed in this work but also detected during the St emulsion polymerization stabilized by SDS alone. ${ }^{25}$ It was found that about $5.8 \%$ of the initially added SDS is adsorbed on the monomer droplet surface in emulsion at CMC and $4.9 \%$ of the initially added SDS is adsorbed at the interface of the water-in-oil (w/o) reverse droplets dispersed inside the monomer droplets. ${ }^{25}$ Thus, the formation of double emulsion droplets decreases the fraction of free monomer and emulsifier initially charged to the polymerization system. Garti and Aserin reported that two stabilizing species are required to prepare multiple emulsion; a relatively hydrophobic emulsifier (e.g., NP 40 ) is used to stabilize the interface of the internal w/o emulsion (reverse emulsion) and a relatively hydrophilic emulsifier (e.g., SDS) is selected to stabilize the interface of the external o/w emulsion (direct emulsion). ${ }^{38}$ The data shown in Figures 3 and 4 can be taken as the supporting evidence that the stability of double emulsion droplets increases with increasing $X$ and the degree of the increased droplet stability is more pronounced beyond ca. $30 \%$ conversion. This is caused by the accumulation of emulsifier (the ratio of emulsifier to monomer in the monomer phase increases with increasing $X$ ) and PSt (hydrophobe) in the monomer phase. Thus, the decreased $R_{\mathrm{p}}$ beyond $c a$. 30-40\% conversion and increased uniformity of emulsion can be connected with the presence of stable double droplets (or the decreased transfer rates of monomer and emulsifier to the reaction loci). The stable monomer droplets are also related to the low radical entry efficiency. ${ }^{32}$

The accumulation of PSt in the monomer phase during the emulsification period $\left(2 \mathrm{~h}\right.$ at $\left.60^{\circ} \mathrm{C}\right)$ and polymerization $(2-4 \mathrm{~h})$ due to the thermally induced polymerization may also increase the stability and uniformity of monomer droplets. ${ }^{39}$ The increased uniformity of monomer emulsion due to the long pre-emulsification period retards the Ostwald ripening effect. The relatively monodisperse monomer emulsion droplets show much higher resistance against the droplet degradation as compared to the polydisperse emulsion of the same recipe ${ }^{40}$ Furthermore, the presence of $c a .0 .5 \mathrm{wt} \%$ predissolved PSt increases the stability of St miniemulsion droplets significantly. ${ }^{41}$ This may be the case for the polymerization system investigated in this work. The thermal polymerization in the monomer droplets may not affect the initial polymerization rate, but it is partially responsible for the increased droplet stability and decreased $R_{\mathrm{p}}$ at medium and high $X$. For example, the monomer conversion achieved by the thermally induced emulsion polymerization of St at $60^{\circ} \mathrm{C}$ is $0.1 \%$ per $\mathrm{h}^{21}$ The initial monomer emulsion contains a large fraction of monomer droplets in the absence of PSt or the concentration of PSt is relatively low to retard the monomer diffusion. Further increasing the PSt concentration in the monomer phase with the progress of polymerization, the diffusion of monomer from the polymer-containing monomer droplets to the reaction loci then becomes restricted.

\section{CONCLUSION}

For the styrene (St) emulsion polymerization, the polymerization rate $\left(R_{\mathrm{p}}\right)$ was independent of the emulsifier charge composition (the ratio of SDS/NP $40=3 / 1,1 / 1$, and $1 / 2$ ) except in the cases of SDS/NP $40=0 / 1$ and $1 / 0$. Smith-Ewart Interval 2 with a constant $R_{\mathrm{p}}$ appears in the narrow conversion range of $20-40 \%$ for the polymerization stabilized by mixed SDS/NP 40 emulsifiers. In the polymerization with SDS alone, non-distinct Interval 2 also occurs in the same conversion range. In the polymerization with NP 40 alone, on the other hand, $R_{\mathrm{p}}$ increases gradually in the course of polymerization. In the polymerizations with SDS alone or mixed SDS/NP 40 emulsifiers, the weight-average diameter of latex particles $\left(d_{\mathrm{w}}\right)$ increases with increasing conversion $(X)$ and the degree of the increased $d_{\mathrm{w}}$ is more pronounced at low $X$. The number of polymer particles per unit volume of water $\left(N_{\mathrm{p}}\right)$ increases strongly with increasing $X$ (up to $20 \%) . N_{\mathrm{p}}$ remains relatively constant when $X$ is greater than $20 \%$. In the polymerization with NP 40 alone, on the other hand, $N_{\mathrm{p}}$ first decreases from the very beginning of polymerization and then levels off. In the polymerization with SDS alone and mixed SDS/NP 40 emulsifiers, the number of radicals per particle $(\bar{n})$ is independent of $X$ except in the very high $X$ region and it reaches a 
value slightly below 0.5 . This is attributed to the closepacked particle surface zone for the system with mixed SDS/NP 40 emulsifiers, which reduces the radical entry and exit rates. The fact that $\bar{n}$ is smaller than 0.5 suggests that the effect of the reduced radical entry rate predominates in the polymerization. The classical mechanism for the entry and exit of radicals governs $\bar{n}$ for the run with SDS alone. By contrast, in the polymerization with NP 40 alone, $\bar{n}$ increases rapidly with increasing $X$ and it reaches a value well above 0.5 . This is interpreted in terms of the coagulative particle nucleation and pseudo-bulk polymerization kinetics. The exit of radicals from the latex particles stabilized by NP 40 is the ratedetermining process. In all polymerizations, the polydispersity index of the particle size distribution is relatively narrow throughout most of the reaction. This is attributed to the very large total particle surface area produced in the early stage of polymerization and the preservation of monomer droplets (the apparent monomerstarved condition). The increased uniformity of the initial monomer emulsion caused by the preparation method adopted in this work shifts the monomer-starved condition to the lower conversion range. The increased stability of monomer droplets results from the accumulation of NP 40, PSt, and inverse micelles in the monomer phase. The continuous release of monomer and emulsifier from monomer droplets is used primarily for the growth and stabilization of latex particles, respectively.

Acknowledgment. The financial support from National Science Council, Taiwan is gratefully acknowledged.

\section{REFERENCES}

1. B. V. Deryagnin and L. D. Landau, Acta Physicochim. USSR, 14, 633 (1941).

2. E. J. W. Verwey and J. Th. G. Overbeek, "Theory of the Stability of Lyophobic Colloids", Elsevier, New York, N.Y., 1943.

3. D. H. Napper, "Polymeric Stabilization of Colloidal Dispersions", Academic Press, London, 1983.

4. M. E. Woods, J. S. Dodge, and I. M. Krieger, J. Paint Technol., 40, 541 (1968).

5. H. Chu and I. Piirma, Polym. Bull., 21, 301 (1989).

6. E. Unzueta and J. Forcada, Polymer, 36, 1045 (1995).

7. J. T. Brooks and M. E. Cates, J. Chem. Phys, 99, 5467 (1993).

8. W. V. Smith and R. W. Ewart, J. Chem. Phys., 16, 592 (1948).

9. I. Capek and L. Q. Tuan, Makromol. Chem., 87, 2063 (1986).

10. I. Capek, J. Barton, L. Q. Tuan, V. Svoboda, and V. Novotny, Makromol. Chem., 188, 1723 (1987).
11. C. S. Chern, S. Y. Lin, L. J. Chen, and S. C. Wu, Polymer, 38, 1977 (1997).

12. C. S. Chern, S. Y. Lin, S. C. Chang, J. Y. Lin, and Y. F. Lin, Polymer, 39, 2281 (1998).

13. L. J. Chen, S. Y. Lin, C. S. Chern, and S. C. Wu, Colloids Surf., A122, 161 (1997).

14. R. G. Gilbert, "Emulsion Polymerization. A Mechanistic Approach", Academic Press, London, 1995.

15. E. Coen, R. A. Lyons, and R. G. Gilbert, Macromolecules, 29, $5128(1996)$.

16. J. M. H. Kusters, D. H. Napper, R. G. Gilbert, and A. L. German, Macromolecules, 25, 7043 (1992).

17. S. Y. Lin, I. Capek, T. J. Hsu, and C. S. Chern, J. Polym. Sci., Polym. Chem. Ed., 37, 4422 (1999).

18. C. S. Chern and Y. C. Liou, Macromol. Chem. Phys., 199, 2051 (1998).

19. H. V. Tartar, J. Phys. Chem., 59, 1195 (1955).

20. P. Becher, J. Colloid Sci., 16, 49 (1961).

21. C. S. Chern, S. Y. Lin, and T. J. Hsu, Polym. J., 31, 516 (1999).

22. J. Brandrup and E. H. Immergut, "Polymer Handbook", 3rd ed, Wiley-Interscience, New York, N.Y., 1981.

23. E. Barthalome, H. Gerrens, R. Herbeck, and H. M. Weitz, Z, Elektrochem., 60, 334 (1956).

24. C. S. Chern and H. Hsu, J. Appl. Polym. Sci., 55, 571 (1995).

25. S. Y. Lin, C. S. Chern, T. J. Hsu, C. T. Hsu, and I. Capek, Polymer, in press.

26. I. Capek and P. Potisk, J. Polym. Sci., Polym. Chem. Ed., 33, 1675 (1995).

27. I. Piirma and M. Chang, J. Polym. Sci., Polym. Chem. Ed., 20, 489 (1982).

28. I. Capek and M. Akashi, J. M. S. Rev. Macromol. Chem. Phys., C33, 369 (1993).

29. H. Schott and S. K. Han, J. Pharm. Sci., 64, 658 (1975).

30. M. I. Gjerde, W. Nerdal, and H. Hoiland, J. Colloid Interface Sci., 183, 285 (1996).

31. E. D. Goddard, Colloids Surf., 19, 255 (1986).

32. J. Reimers and F. J. Schork, J. Appl. Polym. Sci., 59, 1833 (1996); ibid., 60, 251 (1996).

33. Y. T. Choi, M. S. El-Aasser, E. D. Sudol, and J. W. Vanderhoff, J. Polym. Sci. Polym. Chem. Ed., 23, 2973 (1985).

34. J. M. Asua and J. C. De La Cal, J. Polym. Sci., Polym. Chem. Ed., 32, 1761 (1994).

35. E. B. Brandford, J. W. Vanderhoff, and T. Alfrey, J. Colloid Sci., 11, 135 (1956).

36. C. M. Miller, E. D. Sudol, C. A. Silebi, and M. S. El-Aasser, J. Polym. Sci., Polym. Chem. Ed., 33, 1391 (1995).

37. J. Barton and I. Capek, "Radical Polymerization in Disperse Systems", E. Horwood, Ed., Chichester and Veda, Bratislava, 1994.

38. N. Garti and N. Aserin, Adv. Colloid Interface Sci., 65, 37 (1996).

39. I. Capek and C. S. Chern, Adv. Polym. Sci., accepted.

40. J. Bibette, F. L. Calderon, and P. Poulin, Rep. Prog. Phys., 62, 969 (1999).

41. C. M. Miller, E. D. Sudol, and C. A. Silebi, Macromolecules, 28, 2772 (1995). 